\title{
Small interfering RNA targeting Krüppel-like factor 8 inhibits U251 glioblastoma cell growth by inducing apoptosis
}

\author{
WEIFENG WAN, JI ZHU, XIAOCHUAN SUN and WENYUAN TANG \\ Department of Neurosurgery, The First Affiliated Hospital of Chongqing Medical University, Chongqing 400016, P.R. China
}

Received August 10, 2011; Accepted September 30, 2011

DOI: $10.3892 / \mathrm{mmr} .2011 .669$

\begin{abstract}
Small interfering RNAs (siRNAs) are small non-coding RNAs, comprising 21-23 nucleotides that regulate gene expression by transcriptionally repressing their complementary mRNAs. In particular, Krüppel-like factor 8 (KLF8) has been postulated to function as a tumor activator in various cancer cells, but not in glioblastoma. In the present study, we investigated the anti-tumorigenic effect of KLF8 siRNA in glioblastoma cells. U251 human glioblastoma cells were transfected with KLF8 siRNA and assayed for in vitro proliferation, cell cycle and cell apoptosis. The transfection of KLF8 siRNA reduced expression of KLF8 in the glioblastoma cells. KLF8 siRNA also reduced in vitro proliferation and enhanced cell apoptosis. These results indicate that KLF8 siRNA has an anti-tumorigenic effect on glioblastoma cells and suggest the possible use of KLF8 siRNA for the treatment of glioblastoma.
\end{abstract}

\section{Introduction}

Glioblastomas, which are primary brain tumors that arise from glial cells, are the most common primary malignant brain tumors found in adults, and remain formidable despite aggressive surgery, radiotherapy and chemotherapy $(1,2)$. Glioblastomas represent $30-60 \%$ of central nervous system (CNS) primary tumors, with an incidence of 2-3 new cases per 100,000 individuals annually $(3,4)$. Small interfering RNAs (siRNAs) are small, noncoding RNAs, with 21-23 nucleotides, which regulate gene expression by repressing transcription or degrading target mRNA (5). In cancer cells, siRNAs can function as tumor suppressors or oncogenes and, thus, can regulate tumor development and prognosis.

The Krüppel-like factor (KLF) family of transcription factors, which share homology in their three $\mathrm{C} 2-\mathrm{H} 2$ zinc finger

Correspondence to: Dr Ji Zhu, Department of Neurosurgery, The First Affiliated Hospital of Chongqing Medical University, 1 You Yi Road, Chongqing 400016, P.R. China

E-mail: ji_zhu@163.com

Key words: Krüppel-like factor 8, small interfering RNA, glioblastoma, proliferation
DNA binding domains, control diverse processes, including regulation of the cell cycle, proliferation, differentiation, apoptosis, development and tumorigenesis (6-8). KLF8 is a member of the KLF family. Like other members of the KLFs, KLF8 shares the well-conserved DNA-binding zinc finger domains on its C-terminus. The N-terminal portion of KLF8 is thought to determine its functional specificity through the recruitment of other proteins (9). The expression and cellular function of KLF8 is positively regulated by cell signaling molecules, such as Src and PI3K downstream of focal adhesion kinase (FAK) and transcriptional activators including Sp1 and KLF1, and is negatively regulated by transcriptional repression by KLF and post-translational sumoylation (10-13). Overexpressed KLF8 has recently been found in certain human malignant tumors and plays a significant role in oncogenic transformation $(8,14)$. However, whether, and how, KLF8 may play a role in glioblastoma tumorigenesis, invasion and progression is remains unknown.

In the present study, we firstly investigated the expression of KLF8 in glioblastoma cell lines by reverse transcription polymerase chain reaction (RT-PCR). Subsequently, we constructed siRNA sequences targeting KLF8, transfected it into the human glioblastoma cell line, U251, and explored changes in cell growth, cycle and apoptosis.

\section{Materials and methods}

Cell culture. U251, U373, U87 and A172 human glioblastoma cells were obtained from the American Type Culture Collection (Manassas, VA, USA), and cultured in Dulbecco's modified Eagle's medium (DMEM), supplemented with $10 \%$ fetal bovine serum (FBS), $2 \mathrm{mmol} / 1 \mathrm{~L}$-glutamine, $2 \mathrm{mmol} / \mathrm{l}$ sodium pyruvate, 100 units $/ \mathrm{ml}$ penicillin, $100 \mu \mathrm{g} / \mathrm{ml}$ streptomycin and $0.25 \mu \mathrm{g} / \mathrm{ml}$ amphotericin B (all obtained from Invitrogen; Carlsbad, CA, USA).

Construction and transfection of KLF8 siRNA. KLF8 (NM_007250) siRNA or negative control siRNA were inserted into a pGCSIL-green fluorescent protein (GFP) vector (Shanghai Gene Chem). The siRNA plasmids were transfected into 293T cells, together with the lentiviral helper plasmids, pHelper1.0 and pHelper2.0, to generate the respective lentiviruses. Viral stocks were created and used to infect U251 cells. Cells were collected for mRNA level detection, $72 \mathrm{~h}$ following infection. The KLF8 siRNA sequence was 
5'-CAGCACTGTTTAATGACAT-3' and the nonsilencing siRNA sequence was 5'-TTCTCCGAACGTGTCACGT-3'.

RT-PCR and real-time PCR. Total RNA was extracted using TRIzol reagent (Invitrogen) and reverse transcribed using M-MLV-RTase (Promega; Madison, WI, USA), according to the manufacturer's instructions. The resulting complementary DNA (cDNA) was used for real-time PCR using the SYBRGreen Master PCR Mix (Applied Biosystems; Carlsbad, CA, USA) in triplicates. Primers for real-time RT-PCR were as follows: KLF8 sense, 5'-TTCAGAAGGTGGCTCAATGC-3'; KLF8 antisense, 5'-GGAGTGTTGGAGAAGTCATATTAC-3'. PCR and data collection were performed on the TP800 qPCR System (Takara; Japan). All quantifications were normalized to an endogenous glyceraldehyde-3-phosphate dehydrogenase (GAPDH) control. GAPDH sense, 5'-TGACTTCAACAGCG ACACCCA-3'; GAPDH antisense, 5'-GGAGTGTTGGAGAA GTCATATTAC-3'. The relative quantitation value for each target gene compared to the calibrator for that target is expressed as $2^{-(\mathrm{Ct}-\mathrm{Cc})}(\mathrm{Ct}$ and $\mathrm{Cc}$ are the mean threshold cycle differences following normalization to GAPDH).

Cell proliferation assay. Cells infected with KLF8 siRNA or control siRNA for $72 \mathrm{~h}$, respectively, were grown in the exponential phase and detached by trypsin treatment. Viable cells $\left(2 \times 10^{4}\right.$ cells $\left./ \mathrm{ml}\right)$ were plated onto 96-well plates (100 $\mu \mathrm{l} \mathrm{medium/well)} \mathrm{and} \mathrm{cultured} \mathrm{at} 37^{\circ} \mathrm{C}$ in $5 \% \mathrm{CO}_{2}$ atmosphere. Images of 1,000 cells for each treatment were analyzed to obtain the average cell number per field, fluorescence spot number, area and intensity per cell every day at fixed time points using Cellomics instrumentation and software (Thermo Scientific).

BrdU incorporation assay. Cells infected with KLF8 siRNA or the control were cultured in a 96-well plate with 2,000 cells per well. A 5-bromodeoxyuridine (BrdU) incorporation assay was performed using the BrdU cell proliferation assay kit (Chemicon, Temecula, CA, USA). Briefly, $20 \mu 1$ of 1/500 diluted BrdU was added and incubated for $8 \mathrm{~h}$. Following this, $100 \mu \mathrm{l}$ of $1 / 200$ diluted anti-BrdU and peroxidase-conjugated goat anti-mouse $\operatorname{IgG}$ antibodies were used successively, according to the manufacturer's instruction. The plate was washed and $100 \mu 1$ TMB Peroxidase Substrate added. Plates were read at $490 \mathrm{~nm}$.

Cell cycle analysis. For cell cycle analysis, cells infected with KLF8 siRNA or control siRNA for $72 \mathrm{~h}$ were inoculated onto 6 -well plates. Following 3 days of incubation, $5 \times 10^{5}$ cells were harvested, fixed in $70 \%$ ethanol and treated with $100 \mu \mathrm{g} / \mathrm{ml}$ RNase A and $50 \mu \mathrm{g} / \mathrm{ml}$ propidium iodide (PI) in phosphatebuffered saline (PBS) for $15 \mathrm{~min}$ at room temperature in the dark, followed by flow cytometry analysis using FACScalibur flow cytometer (BD Pharmingen, San Diego, CA, USA).

Cell apoptosis assay. To detect apoptotic cells, the cells infected with KLF8 siRNA or control siRNA for $72 \mathrm{~h}$ were inoculated into 6 -well plates. Following 3 days of incubation, cells were harvested and stained with Annexin V-allophycocyanin (APC), using an apoptosis detection kit (eBioscience), according to the manufacturer's instructions.
A

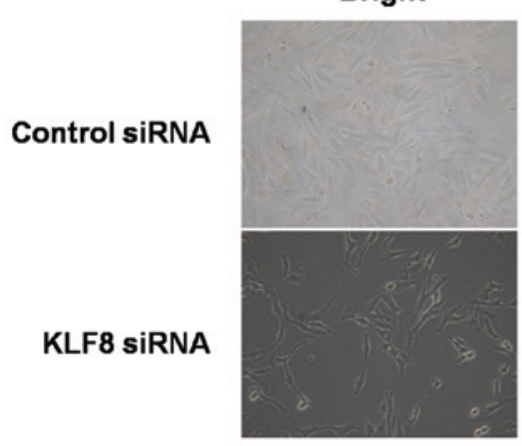

Bright Fluorescent

B

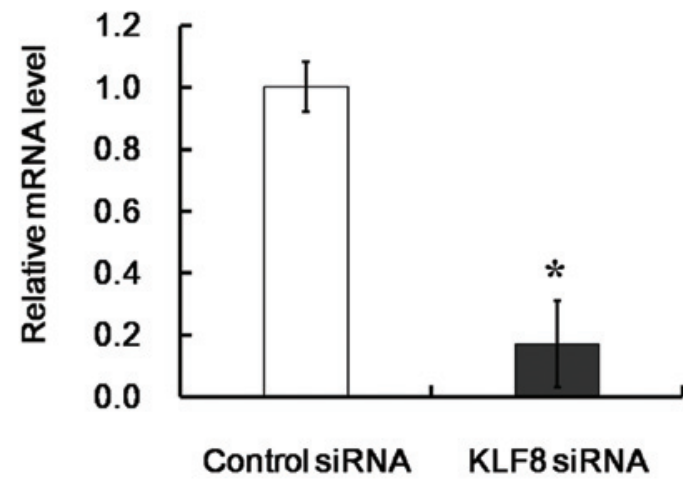

Figure 1. RNAi led to the down-regulation of KLF8 in the glioblastoma cell line, U251. Control siRNA and KLF8 siRNA lentivirus were infected into U251 cells. Micrographs were taken $72 \mathrm{~h}$ following infection (magnification, $\mathrm{x} 200$ ). (B) The mRNA level of KLF8 was down-regulated by KLF8 siRNA in U251 cells ( $\left.{ }^{*} \mathrm{P}<0.05\right)$. siRNA, small interfering RNA; KLF8, Krüppel-like factor 8; RNAi, RNA interference.

Samples were then analyzed by FACScalibur flow cytometer (BD Pharmingen).

Statistical analysis. All experiments were repeated three times with triplicate samples. Student's t-test was used to compare the values of the test and control samples. A value of $\mathrm{P}<0.05$ was considered to be statistically significant.

\section{Results}

KLF8 siRNA down-regulates the expression of KLF8 in human glioblastoma cell line U251. KLF8 mRNA expression in U87, U251, A172 and U373 cells was analyzed by semi-quantitative real-time PCR and agarose gel electrophoresis. GAPDH served as the control. All four human glioblastoma cell lines expressed KLF8 mRNA (data not shown).

The recombinated lentiviruses, expressing siRNA or negative control siRNA, were infected into human glioblastoma cell line U251 cells (Fig. 1A). At a multiplicity of infection (m.o.i.) of 10, the infection efficiency was over $80 \%$, as determined by FACS analysis for GFP expression. To determine the effect of RNA interference (RNAi) on the expression of KLF8 in U251 cells, the mRNA levels of KLF8 were analyzed. The cells infected with KLF8 siRNA exhibited lower expression of KLF8 mRNA than those with negative control siRNA (Fig. 1B).

KLF8 SiRNA reduces the proliferation of human glioblastoma cells. We then investigated the effect of KLF8 siRNA 
A

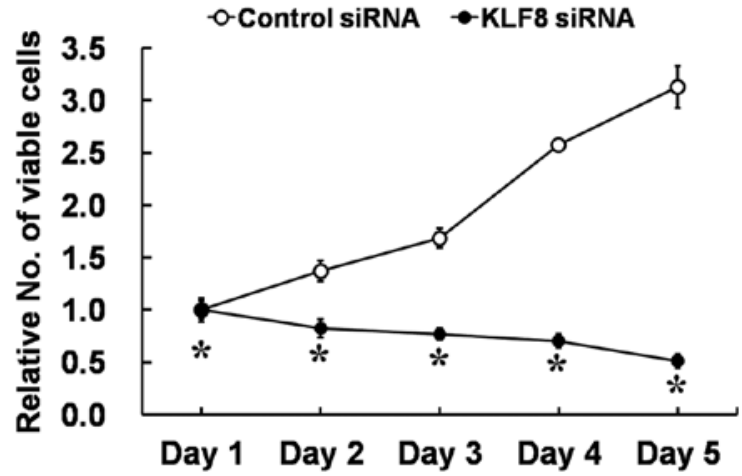

B

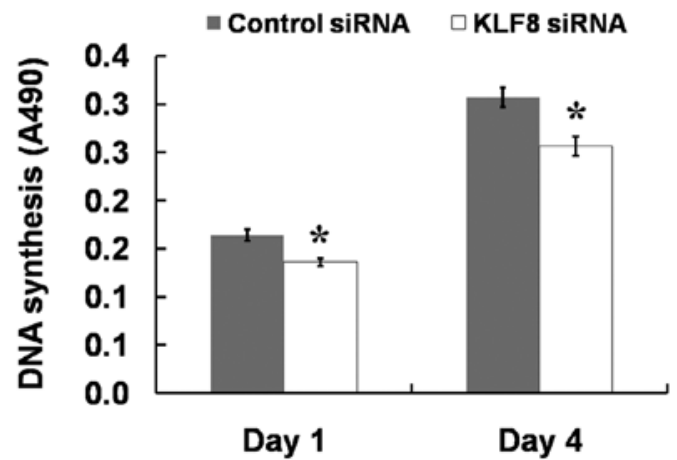

Figure 2. The growth of glioblastoma U251 cells was inhibited upon KLF8 siRNA treatment. (A) Cell number was counted using Cellomics Arrayscan following 1, 2, 3, 4 and 5 days of incubation, and the increased fold in cell number was calculated. (B) The BrdU incorporation of cells was examined following 1 and 4 days of incubation. Mean values are derived from three independent experiments, errors indicate standard deviation. ${ }^{*} \mathrm{P}<0.05$. siRNA, small interfering RNA; KLF8, Krüppel-like factor 8.
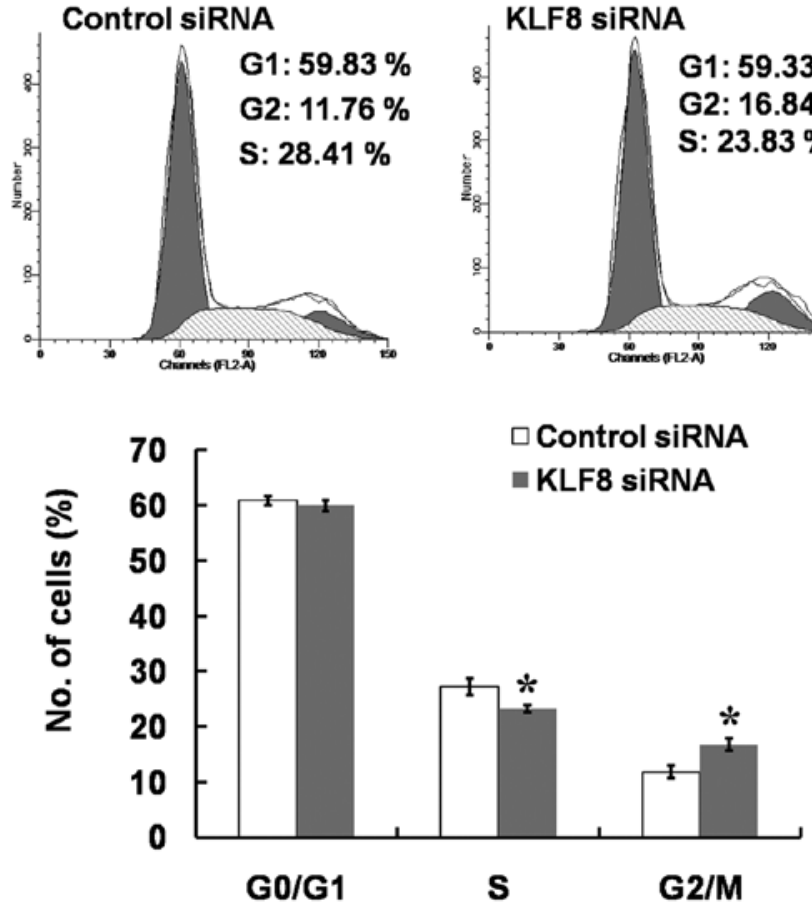

Figure 3. KLF8 siRNA affected the cell cycle of U251 cells. The cell cycle distribution profile indicates that, following KLF8 inhibition, U251 cells were arrested in the second gap (G2) phase of the cell cycle (upper panel). Statistical results of the population of cells in each cell cycle (lower panel). Mean values are derived from three independent experiments, errors indicate standard deviation. ${ }^{\mathrm{P}}<0.05$. siRNA, small interfering RNA; KLF8, Krüppel-like factor 8.
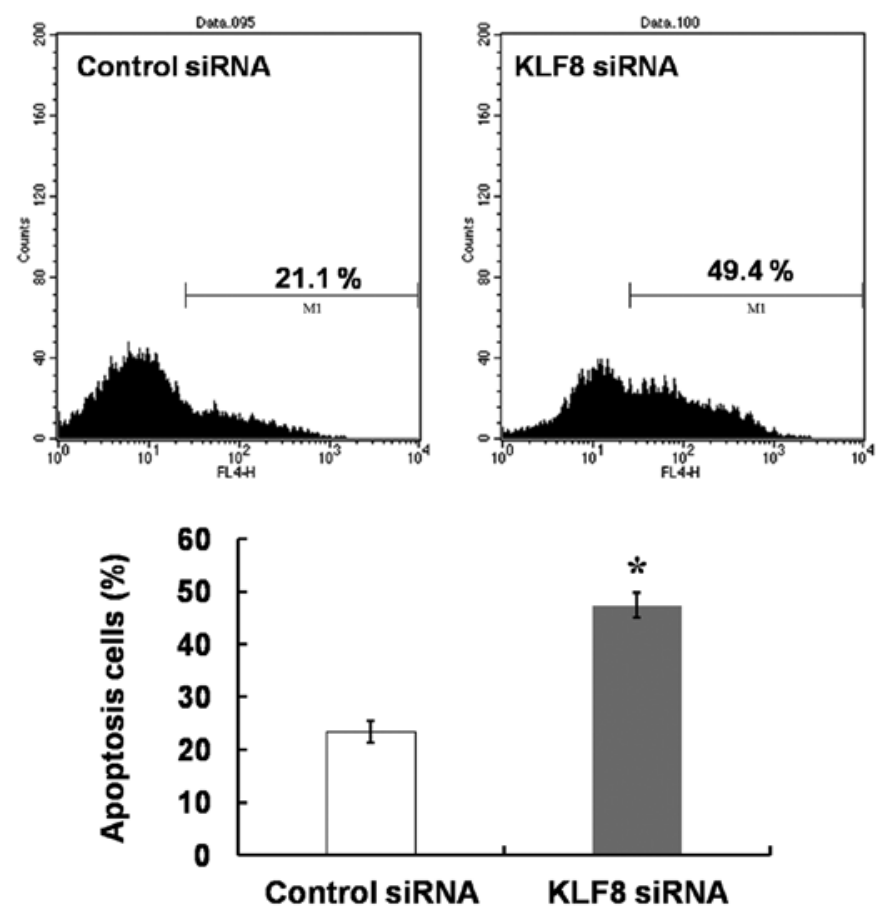

Figure 4. KLF8 siRNA affected the cell cycle of U251 cells. Histogram of cell apoptosis (upper panel). The cell apoptosis assay revealed increased apoptosis of cells when treated with KLF8 siRNA (lower panel). Mean values are derived from three independent experiments, errors indicate standard deviation. ${ }^{*} \mathrm{P}<0.05$. siRNA, small interfering RNA; KLF8, Krüppel-like factor 8 .

on glioblastoma cell proliferation. As shown in Fig. 2A, the growth pattern observed changed visibly among transfectants from the second to the fifth day in U251 cells. Cells treated with KLF8 siRNA exhibited lower growth rates compared with the negative control siRNA. The results revealed that the knock-down of KLF8 by siRNA in glioblastoma cells did have an inhibitory effect on cell growth and proliferation. Moreover, BrdU incorporation assay also revealed that the inhibition of KLF8 expression significantly reduced the growth rate of U251 cells during the 4-day incubation period $(\mathrm{P}<0.05$ compared with the control, Fig. $2 \mathrm{~B})$. These findings sustain the notion that the knock-down of KLF8 greatly diminishes the cell proliferative ability of U251 cells.

KLF8 siRNA affects the cell cycle and enhanced the apoptosis of human glioblastoma cells. We performed a cell cycle assay to detect whether KLF8 siRNA affected U251 cell cycle by FACScalibur flow cytometer $72 \mathrm{~h}$ following infection. The results revealed that there was notable change in cell cycle phase distributions between U251 cells treated with KLF8 siRNA and control siRNA (Fig. 3). It was suggested that KLF8 siRNA caused G2/M phase arrest and affected cell cycle progress. We then explored whether KLF8 siRNA affected U251 cell apoptosis. The results revealed that KLF8 siRNA enhanced the rate of apoptosis (Fig. 4). These results suggest that KLF8 siRNA inhibited glioblastoma cell proliferation through the enhancement of cell apoptosis. 


\section{Discussion}

In the present study, we investigated the effect of KLF8 siRNA infection in glioblastoma cells by quantifying cellular proliferation and apoptosis. Our results suggest that KLF8 siRNA has anti-tumorigenic effects on glioblastoma cells, which may be attributed to reduced cell proliferation and the enhanced cell apoptosis.

Aberrant overexpression of KLF8 has been found in numerous human cancer cell types, including ovarian and breast cancer, and plays a critical role in oncogenic transformation, epithelial to mesenchymal transition and cancer cell invasion. In addition, KLF4 and E-cadherin tumor suppressors have been identified as KLF8-repressed targets $(8,10,14)$. KLF8 has thus emerged as one of the most important oncogenic transcription factors (15). The expression, transcriptional activity and nuclear localization of such transcription factors are tightly regulated in normal cells, and when dysregulation occurs the cells go awry. However, the underlying mechanism that results in KLF8 over-activation is currently unknown. A previous study demonstrated that KLF8 mediated cell cycle progression downstream of FAK by up-regulating cyclin D1 (7), and that the expression of wild-type FAK resulted in enhanced expression of KLF8 in glioblastoma cells (12). Further studies are required to explore the detailed mechanism.

Despite numerous studies with varying treatment modalities, glioblastoma remains highly resistant to systemic therapy $(1,2)$. It is important to discover a new way to effectively inhibit glioblastoma cell growth and avoid the side effects of drugs. Gene target therapies have proven to be a promising way to achieve this goal (16-18). siRNA was constructed as a promising new approach for gene silencing in cells over the years $(19,20)$. This inhibits gene expression in a sequence-specific manner by degradation of the corresponding mRNA and has been proven to be a powerful tool for suppressing gene expression (21-23). siRNA is now widely used in cancer studies and may provide a promising new approach towards glioblastoma therapy. Therefore, our results suggest that KLF8 siRNA may be used as a potent tool in glioblastoma therapy.

\section{References}

1. Voelzke WR, Petty WJ and Lesser GJ: Targeting the epidermal growth factor receptor in high-grade astrocytomas. Curr Treat Opt Oncol 9: 23-31, 2008.

2. Ohgaki H and Kleihues P: Epidemiology and etiology of gliomas. Acta Neuropathol 109: 93-108, 2005.

3. Wrensch M, Minn Y, Chew T, et al: Epidemiology of primary brain tumors: current concepts and review of the literature. Neuro Oncol 4: 278-299, 2002.
4. Schwartzbaum JA, Fisher JL, Aldape KD, et al: Epidemiology and molecular pathology of glioma. Nat Clin Pract Neurol 2: 494-503, 2006.

5. Elbashir S, Harborth J, Lendeckel W, et al: Duplexes of 21-nucleotide RNAs mediate RNA interference in cultured mammalian cells. Nature 411: 494-498, 2001.

6. Pearson R, Fleetwood J, Eaton S, et al: Krüppel-like transcription factors: a functional family. Int J Biochem Cell Biol 40: 1996-2001, 2008.

7. Zhao J, Bian ZC, Yee K, et al: Identification of transcription factor KLF8 as a downstream target of focal adhesion kinase in its regulation of cyclin D1 and cell cycle progression. Mol Cell 11: 1503-1515, 2003.

8. Wang $X$ and Zhao J: KLF8 transcription factor participates in oncogenic transformation. Oncogene 26: 456-461, 2007.

9. Quadrini KJ and Bieker JJ: Krüppel-like zinc fingers bind to nuclear import proteins and are required for efficient nuclear localization of erythroid Krüppel-like factor. J Biol Chem 277: 32243-32252, 2002.

10. Wang X, Urvalek AM, Liu J, et al: Activation of KLF8 transcription by focal adhesion kinase in human ovarian epithelial and cancer cells. J Biol Chem 283: 13934-13942, 2008.

11. Wei H, Wang X, Gan B, et al: Sumoylation delimits KLF8 transcriptional activity associated with the cell cycle regulation. J Biol Chem 281: 16664-16671, 2006.

12. Ding Q, Grammer JR, Nelson MA, et al: p27Kip1 and cyclin D1 are necessary for focal adhesion kinase regulation of cell cycle progression in glioblastoma cells propagated in vitro and in vivo in the scid mouse brain. J Biol Chem 280: 6802-6815, 2005.

13. Eaton SA, Funnell AP, Sue N, et al: A network of Kruppel-like Factors (Klfs). Klf8 is repressed by Klf3 and activated by Klf1 in vivo. J Biol Chem 283: 26937-26947, 2008.

14. Wang X, Zheng M, Liu G, et al: Krüppel-like factor 8 induces epithelial to mesenchymal transition and epithelial cell invasion. Cancer Res 67: 7184-7193, 2007.

15. Wang $X$ and Zhao J: KLF8 transcription factor participates in oncogenic transformation. Oncogene 26: 456-461, 2007.

16. Imyanitov EN and Moiseyenko VM: Molecular-based choice of cancer therapy: realities and expectations. Clin Chim Acta 379: $1-13,2007$.

17. Masiero M, Nardo G, Indraccolo S, et al: RNA interference: implications for cancer treatment. Mol Aspects Med 28: 143-166, 2007.

18. Tschoep K, Kohlmann A, Schlemmer M, et al: Gene expression profiling in sarcomas. Crit Rev Oncol Hematol 63: 111-124, 2007.

19. Elbashir SM, Harborth J, Lendeckel W, et al: Duplexes of 21-nucleotide RNAs mediate RNA interference in cultured mammalian cells. Nature 411: 494-498, 2001.

20. McManus MT and Sharp PA: Gene silencing in mammals by small interfering RNAs. Nat Rev Genet 3: 737-747, 2002.

21. Mandriota SJ, Jussila L, Jeltsch M, et al: Vascular endothelial growth factor-C-mediated lymphangiogenesis promotes tumour metastasis. EMBO J 20: 672-682, 2001.

22. Padera TP, Kadambi A, di Tomaso E, et al: Lymphatic metastasis in the absence of functional intratumor lymphatics. Science 296: 1883-1886, 2002.

23. Rocchi P, Jugpal P, So A, et al: Small interference RNA targeting heat-shock protein 27 inhibits the growth of prostatic cell lines and induces apoptosis via caspase-3 activation in vitro. BJU Int 98: 1082-1089, 2006. 